The book appears to be addressed to the initiated; no concessions are made to those unfamiliar with the concepts used. It would be ideal for someone who has had two or three years away from human or medical genetics and who wants a rapid update (significant advances since the date of the conference in knowledge of the dystrophin gene and of the cystic fibrosis region are covered by notes added in proof). It can also be recommended to trainees in medical genetics from either a clinical or laboratory background, especially if they use it as a key to recent publications. Given its rather limited appeal, however, I am unconvinced of the justification for publishing the proceedings of what was undoubtedly a valuable meeting.

\section{N R DENNIS}

\section{Advances in Human Genetics}

Volume 17. Edited by Harry Harris and Kurt Hirschhorn. (Pp 209; £49.50.) New York, London: Plenum Press. 1988.

Since the first volume appeared in 1970, this series has established itself as authoritative reviews on all aspects of human genetics. However, because the subjects covered have on occasions been somewhat specialised, some volumes may have been outside the immediate interests of any individual medical geneticist. This is not so with this recent volume for four of the five subjects covered will be of interest to most practising geneticists. The one exception is a chapter on human salivary proteins and their polymorphisms which may have restricted appeal but is nevertheless a scholarly and detailed review. The remaining four chapters deal with chorionic villus sampling, the molecular genetics of the haemophilias, cloning of the Duchenne/Becker muscular dystrophy locus, and molecular and recent cytogenetic studies in Down's syndrome.

Chorionic villus sampling has become an established procedure for the prenatal diagnosis of most genetic disorders and worldwide analysis of some 20000 cases now indicates that the risks of the procedure are very small. The applications in? chromosome, metabolic, and DNA analyses are discussed. It seems clear that investigators are often? not satisfied with direct methods of analysis ande short term culture of villus material is preferred. It is also clear that in the case of metabolic disordersthere have been discrepancies in the diagnostio evaluation in several cases which emphasises theis importance of experienced laboratory facilities in $-\overrightarrow{0}$ this area.

Details of the range of molecular defects in the $\overrightarrow{\vec{\omega}}$ haemophilia A gene (186 kb with 26 exons) and the haemophilia B gene (34 kb with eight exons) are being revealed. However, the relationship of dele $\dot{i}$ tions to the presence of inhibitors to F VIII and F IX? is still not understood and so far no clear picture has emerged concerning the association of inhibitoro formation and the size and position of deletions.o The identification of DNA polymorphisms within the F VIII and F IX genes has revolutionalised carrier detection and prenatal diagnosis in these disorders.

The story of the localisation of the Duchenne/\% Becker gene and the subsequent isolation of its product is well surveyed by Monaco and Kunkel, who have contributed so much to this field. Aston-s ishingly much of this brilliant work by the firsto author represented requirements for a $\mathrm{PhD}$ ato Harvard! To quote the poet W B Yeats:

All his twenties crammed with toil; “What then?" sang Plato's ghost. "What then?’3

In our onward rush to study molecular genetics we perhaps should remember that many other problems in human genetics still remain to be solved, and this is nowhere more evident than in Down's syndrome. The authors of this review show how the new cytogenetic and molecular studies mayo provide helpful information on such matters as the origin of non-disjunction, how maternal age is relevant, and the possible effects on the phenotype of the parental origin of trisomy.

There is much to interest most medical geneticists: in this volume. 